\title{
Calculations on a Class of Candidate Solutions for the Distortion Problem on $\ell_{2}$
}

\author{
Frank Sanacory* \\ Department of Mathematics and CIS, SUNY College at Old Westbury, Old Westbury, NY 11568 \\ sanacoryf@oldwestbury. edu
}

March 16, 2019

\begin{abstract}
In 1994 E. Odell and Th. Schlumprecht showed that the Hilbert space $\left(\ell_{2}\right)$ was arbitrarily distortable. However, we do not have a defined sequence of norms that would perform this distortion. G. Androulakis and the author defined a class of norms equivalent to the Hilbert space norm. These norms are candidate sequences of norms that might distort $\ell_{2}$. These norms are recursively defined and calculations with these norms are quite difficult. Here we present a tool, a Python program, that calculates the norms defined by G. Androulakis and the author.
\end{abstract}

Keywords Distortion · Banach Spaces · MSC 2010: 46B25, 46C15, 68

\section{Introduction to the Distortion Problem}

In the paper [1] a family of some new Tsirelson-type norms were introduced which are equivalent to usual norm on the separable Hilbert space $\ell_{2}$. The motivation for presenting these norms is the following question of E. Odell and Th. Schlumprecht [3] which is also mentioned by T.W. Gowers [2]:

The Distortion Problem. Is it possible, for every $\lambda>0$ to explicitly define an equivalent norm $\|\cdot\|$ on $\ell_{2}$ such that every infinite dimensional subspace $Y$ of $\ell_{2}$ contains two vectors $y_{1}$ and $y_{2}$ with with $\left\|y_{1}\right\|_{2}=\left\|y_{2}\right\|_{2}=1$ (where $\|\cdot\|_{2}$ denotes the usual norm of $\ell_{2}$ ) and $\left|y_{1}\right| /\left|y_{2}\right|>\lambda$ ?

It is known that there exists some sequence of norms to satisfy the distortion problem [3, 4]. However, there is no explicitly defined solution. That is, we know the distortion problem has the answer yes, but we do not know the sequence of norms. This problem has been open for nearly 30 years and there has been some work done. Androulakis and Sanacory presented a candidate sequence of norms to answer the question (see [1]). However, calculations are too complicated and present difficulties in showing distortion on this new norm.

\section{Candidates to solve the Distortion Problem}

To define the new norms equivalent to the $\ell_{2}$-norm on $\ell_{2}$ we first introduce some notation. For $x=(x(i)) \in \ell_{2}$ and $E \subseteq N$ we denote by $E x$ the natural projection of $x$ on $E$, i.e. $E x=((E x)(i))$ where $(E x)(i)=x(i)$ for all $i \in E$ and $(E x)(i)=0$ otherwise. Let $c_{00}$ be the vector space of scalar sequences with finite support. Set

$$
\mathcal{A}=\left\{y=(y(i))_{i=1}^{N} \in(0, \infty)^{N}: N \in \mathbb{N}, N \geq 2, \sum_{i=1}^{N}|y(i)|^{2}=1\right\}
$$

We define a sequence of equivalent norms $\|\cdot\|_{y}$, for $y \in \mathcal{A}$, on $\ell_{2}$ as follows. Fix $y=(y(i))_{i=1}^{N} \in A$. Then $\|\cdot\|_{y}$ is the unique norm which satisfies

$$
\|x\|_{y}=\sup \sum_{i=1}^{N} y(i)\left\|E_{i} x\right\|_{y} \vee\|x\|_{\infty} \text { for every } x \in c_{00},
$$

wWw . sanacory . net 


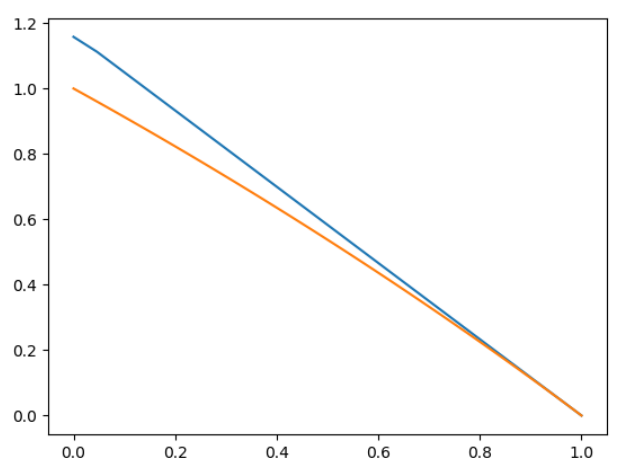

Figure 1: The horizontal axis shows the two vectors have the same norm while the vertical axis shows the vectors norms deviate.

where the supremum is taken with respect to any sequence of sets $E_{1}<E_{2}<\cdots<E_{N}$. Notice that in the definition of $\|\cdot\|_{y}$ we allow the sets $E_{i}$ to be empty and we adopt the convention that " $A<\emptyset$ " and " $\emptyset<B$ " are valid for any $A, B \subseteq N$. We now show that for every $y \in \mathcal{A}$ there exists a unique norm $\|\cdot\|_{y}$ which satisfies 2

For every fixed $y \in \mathcal{A}$ we define a sequence of norms $\|\cdot\|_{y}^{(n)}$ for $n \in \mathbb{N} \cup\{0\}$ which increases to the norm $\|\cdot\|_{y}$, as follows. Let $\|\cdot\|_{y}^{(0)}=\|\cdot\|_{\infty}$ (the $\ell_{\infty}$ norm). If for $n \in \mathbb{N} \cup\{0\}$ the $\|\cdot\|_{y}^{(n)}$ has been defined, then we define

$$
\left.\left.\|x\|_{y}^{(n+1)}=\sup \sum_{i=1}^{N} y(i)\|E i x\|_{y}^{(} n\right) \vee\|x\|_{y}^{(} n\right) \text { for } x \in c_{00},
$$

where the supremum is taken with respect to any sequence of sets $E_{1}<E_{2}<\cdots<E_{N}$.

Then $\|\cdot\|_{y}$ is the (pointwise) limit of $\|\cdot\|_{y}^{(n)}$ for $n \in \mathbb{N} \cup\{0\}$, and it satisfies 2

\section{Calculations}

The calculations are very difficult by hand. Se we used computer power to write a program in Python. The program will compute the norm of one vector or the norm of a net of vectors of a given dimension. The program can be found at https://github.com/sanacoryf/ASNorm. Since the definition of the AS norms are recursive, the calculation can take some time 2 as the dimension of the calculation increases.

\subsection{One Sample Calculation}

The program can calculate the norm (given some norming vector referred to as $y$ above) of a single vector or it can calculate the norm of a net (with an adjustable delta of 0.1 units) of vectors in the positive orthant of some given dimension $\mathrm{n}$.

We will run an example running the calculation over a net. So to perform a calculation we will need a norming vector (or a family of norming vectors) and the dimension of the net.

For our sample calculation we will look at a family of vectors in the sphere of $\ell_{2}$. Let's look at the family $y_{n}=$ $(1 / \sqrt{n})_{i=1}^{n}$ for $n>2$. So our first vector for consideration would be $y_{2}=(1 / \sqrt{2}, 1 / \sqrt{2})$. And we will compute over dimension $n=4$.

We get the out put in the file norms.txt.

$\begin{array}{lrr}\text { vector } & (1.0,1.0,1.0, & 1.0) \\ \text { ASNorm } & & 2 \\ \text { ENorm } & & 2 \\ \text { Ratio } & 1 \\ \text { Name: } 1330, \text { dtype: object } \\ \text { vector } \quad(0.4,1.0,0.2,0.4) \\ \text { ASNorm } & 1.00711 \\ \text { ENorm } & 1.16619 \\ \text { Ratio } & 0.863587 \\ \text { Name: } 1841, \text { dtype: object }\end{array}$

\footnotetext{
${ }^{2}$ The calculations were run on single linux machine with an i7 8th generation chip and 32 GB RAM.
} 
Giving a pair of vectors that maximizes the ratio $\left|y_{1}\right| /\left|y_{2}\right| \approx 1 / 0.863587 \approx 1.158$. Not very much distortion yet. The program also outputs a graph in the slice of the unit ball defined by the span of $y_{1}$ and $y_{2}$ and displays the $\ell_{2}$ norms of $y_{1}$ and $y_{2}$ normalized in $\ell_{2}$. So their $\ell_{2}$ norms are one but their $\|\cdot\|_{y}$ norms display the distortion.

\section{References}

[1] Androulakis, G., \& Sanacory, F. (2007). Banach Spaces and their Applications in Analysis, 241-250 de Gruyter 2007. In Banach Spaces and Their Applications in Analysis: Proceedings of the International Conference at Miami University, May 22-27, 2006, in Honor of Nigel Kalton's 60th Birthday. Walter de Gruyter.

[2] Gowers, W.T. Ramsey. Methods in Banach spaces. Handbook of the geometry of Banach spaces, Vol. 2, 1071-1097, North-Holland, Amsterdam, 2003.

[3] Odell, E.; Schlumprecht, Th. The distortion problem. Acta Math. 173 (1994), no. 2, 259-281.

[4] Odell, E. ; Schlumprecht, Th. Distortion and stabilized structure in Banach spaces; new geometric phenomena for Banach and Hilbert spaces. Proceedings of the International Congress of Mathematicians, Vol. 1, 2 (Zürich, 1994), 955-965, Birkhäuser, Basel, 1995. 